\title{
Summary
}

This paper is a review of 102 cases of intra-ocular foreign bodies evacuated to one of the hospitals serving the Eighth Army in its North African Campaign from November, 1941, to May, 1943.

An analysis is made of the type of casualty sustained, the nature of the missile, the site of penetration, intra-ocular complications and the incidence of multiple wounds. About 30 per cent. of war missiles have low magnetic properties. When these are situated behind the lens the posterior route of extraction through a scleral incision is the method of choice. The technique of this operation is described in detail.

Visual results and complications are discussed.

I express my thanks to Brigadier G. I. Scott, Consultant Ophthalmologist, M.E. and to Colonel H. D. F. Brand, Officer Commanding, General Hospital, M.E.F., for permission to publish this paper. Also to Major W. H. Milligan for much helpful advice and pleasant co-operation in radiographic matters ; to Major G. C. Dansey Browning for his thorough account of the military itinerary of No. 1 Mobile Ophthalmic Unit and for his kindness in showing me the conditions of his work as a field eye surgeon; to Capt. E. J. Hewett, R.E., for' supplying the map prints of North Africa, and lastly to $\mathrm{Cpl}$. McNicol for his photographic work on the foreign bodies.

\section{ORBITAL CELLULITIS IN A BABY CAUSED BY ACUTE OSTEOMYELITIS OF THE MAXILLA*}

BY

\author{
JOHN A. MAGNUS \\ YOR K
}

IN 1939 Asherson published a paper entitled " Acute Osteomyelitis of the Superior Maxilla in Young Infants." In it he reviews the whole literature, comprising; together with his own, 42 cases of infants under twelve weeks of age.

He draws attention to the fact that orbital cellulitis in young. children, which used to be regarded as being caused by an acute inflammation of the antrum, is in reality an osteomyelitis of the maxilla, with secondary involvement of the antrum, because it is characterized by the formation of fistulae and sequestra.

He shows that the staphylococcal infection is in the maxillary bone, involving the tooth socket of the upper deciduous, unerrupted, first molar. The socket is at this age actually larger than the antrum, to which it is closely related. Any infection of 
it involves, therefore, the antrum, and spreads to the orbit, canine fossa, and palate, forming multiple abscesses and fistulae.

The external fistula is always in the same situation, namely, in the medial part of the lower eyelid. The source of the infection may be the mother's genital tract, or an ulcerated nipple. It may come from the nurse or doctor in attendance, or from boils or an infected umbilicus of the child himself.

Asherson summarizes the differential diagnosis between osteor myelitis of the superior maxilla and orbital cellulitis due to nasal sinusitis as follows :-

Osteomyelitis of the Superior Maxilla

(1) Age of infant. Invariably within the first 12 weeks of life

(2) Organism, staphylococcus

(3) Swelling of both eyelids. Swelling of canine fossa. Swelling of hard palate

(4) Proptosis, chemosis and ophthalmoplegia

(5) Fistula in the alveolus. Swelling in the alveolus

(6) Sequestrum formation. Tooth may be extruded

(7) Persistent facial or alveolar fistula. Palatal fistula

(8) Response to operation; repeated relapses common.

Requires prolonged postoperative observation for years

(9) Situation of the abscess. On the floor of the orbit, nearer the outer wall

(10) Nasal discharge, usually unilateral, and late in onset. Not an initial symptom. Staphylococcus cultured

(11) Operation unavoidable. External drainage necessary
Orbital Involvement secondary to Acute Nasal Sinusitis. (Orbital Cellulitis)

After 6 months, but ary age

\section{Streptococcus}

Swelling of eyelids only

Proptosis, chemosis, and ophthalmoplegia

None

None

None. Facial wound invariably heals up

Good. Usually heals up rapidly with good drainage through the nose

Breakdown of the wound uncommon

In the infant, at the junction of the medial orbit wall and the floor. In older children and adults at the junction of the medial wall and the roof

Nasal discharge, bilateral; an early and usually initial symptom.

Streptococcùs cultured

Operation may be avoided in early cases. External drainage may be avoided 
The treatment recommended for osteomyelitis of the superior maxilla is :-

The immediate drainage of the orbital abscess by an external incision through the medial part of the lower lid. If necessary counter drainage through the medial wall of the orbit into the nose.

The abscesses in the canine fossa and alveolus are incised through the mouth.

In addition to the surgical treatment, sulphathiazole should be given, and special attention be paid to the feeding of the baby. Mortality is about 25 per cent.

The case in question is Susan W., aged two weeks. She was admitted to hospital on December 24, 1942.

The mother said that the infant had had a cold and nasal catarrh with nasal discharge. The left eye started to get swollen two days ago.

\section{Condition on Admission}

Marked oedema of the left upper and lower lid, chemosis and proptosis of the eyeball.

The cornea appeared to be normal.

There was a haemorrhagic mucous discharge from the left nostril.

Temperature-100.8 .

The nasal discharge was examined for diphtheria, and proved to be negative.

Hot fomentations were applied to the eye, and guttae arg. nitr., $\frac{1}{2}$ per cent., to the nose.

The baby was fed by her mother.

Within the next two days the proptosis increased, and the clinical picture developed into a typical orbital cellulitis.

The baby was now put on M. \& B. 693 (first dose $\frac{1}{2}$ tablet, followed by $\frac{1}{4}$ tablet four-hourly) and compresses of sodium sulphate solution, 12 per cent., to the left eye.

Mr. R. Thomas, the Ear, Nose and Throat Specialist, was consulted, and he suggested that it might be a case of osteomyelitis of the maxilla.

On the fifth day after admission he operated on the child. A deep incision was made through the medial part of the left lower lid down to the bone, which felt rough. A large sub-periosteal abscess in the floor of the orbit was opened, and excessive pus discharged from the opening, which was kept. open by, drainage (see Fig. 1).

The compresses with sodium sulphate 12 per cent., were continued, and a suction apparatus was used frequently to clear out the discharge from the nose and incision wound. The proptosis and swelling of the lids subsided within the next four days. There was abundant purulent discharge from the incision wound and the left nostril. The temperature returned to normal. 


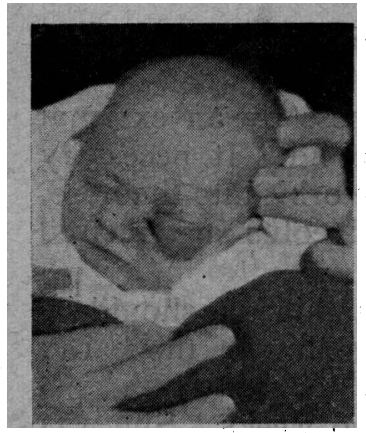

FIG. 1 .

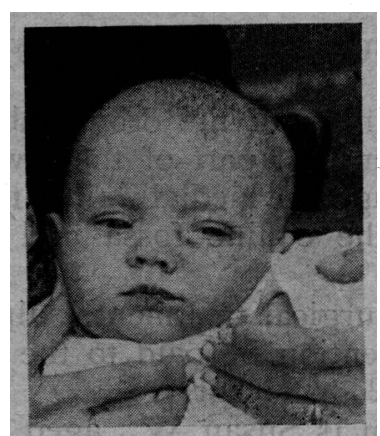

FIG. 2.

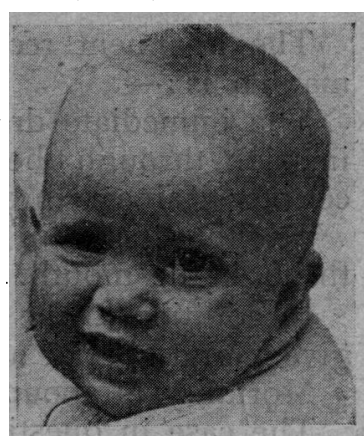

FIG. 3.

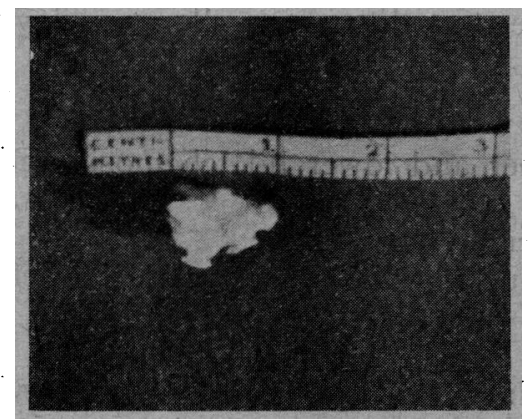

FIG. 4.

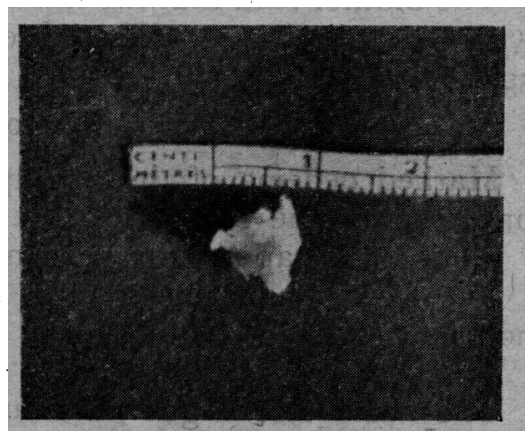

FIG. 5

Ten days after the operation the left lower lid started to swell again, and an abscess formed in the outer corner, which burst spontaneously. During the next two months the condition improved satisfactorily.

The left eye returned to normal, and the incision in the left lower lid closed, except for a small fistula, which discharged slightly (Fig. 2). The baby's weight had increased steadily from $6 \mathrm{lb} .6 \mathrm{oz}$. on admission to $9 . \mathrm{lb} .14 \mathrm{oz}$. when discharged from hospital on March 5, 1943.

In April, 1943, a sequestrum (8 millimetres x 10 millimetres in size, see Figs. Nos. 4 and 5) appeared in the fistula of the lower lid, and was removed with forceps.

The fistula very soon closed, and the further progress was uneventful.

When last seen in September, 1943 (nine months after the operation), the scar of the left lower lid was scarcely visible, and the child was in perfect-health (see Fig. 3):

My thanks are due to Mr. R. Thomas, Honorary Ear, Nose and Throat Surgeon to the York County Hospital, for his kind help and advice in this case.

\section{REFERENCES}

Asherson, N. (1939).- "Acute osteomyelitis of the superior maxilla in young infants." Jl. of Larygnol., Vol. LIV, p. 691.

BARKER, G. M. (1942).-Jl. of Laryngol. and Otol., Vol. LXII, p. 157. 